\title{
Estimating residential energy flexibility potential through forced activations
}

\author{
Hussain Kazmi \\ Dept. of Electrical Engineering \\ KU Leuven, Belgium \\ hussainsyed.kazmi@kuleuven.be
}

\author{
Pieterjan Vaneerdewegh \\ Dept. of Electrical Engineering \\ KU Leuven, Belgium
}

\author{
Johan Driesen \\ Dept. of Electrical Engineering \\ KU Leuven, Belgium
}

\begin{abstract}
Energy flexibility characterization studies have largely focused on the case of smart buildings that respond to penalty or activation signals. This assumes the presence of flexible loads or human occupants in the building which modulate their behavior according to a penalty signal. In practice, defining and implementing this penalty signal is challenging in the residential context due to limited economic rewards and low price elasticity of householders. However, in many developing countries, electricity grid outages or black-outs are endemic, and it is possible to view these blackouts as recurring 'forced' flexibility activation (or very large penalty) signals. In this paper, we study the response of 42 residential buildings in Pakistan to these activation signals to better understand the energy flexibility potential of ubiquitous UPS systems. Utilizing real-world demand data, the paper also demonstrates a method to estimate the amount of curtailed and shifted energy. Understanding and leveraging this flexibility will play a crucial role in enabling future demand response programs in developing countries.
\end{abstract}

\section{KEYWORDS}

Demand side management, energy flexibility, forecasting, Pakistan, battery-inverter systems.

\section{ACM Reference Format:}

Hussain Kazmi, Pieterjan Vaneerdewegh, and Johan Driesen. 2021. Estimating residential energy flexibility potential through forced activations. In The 8th ACM International Conference on Systems for Energy-Efficient Buildings, Cities, and Transportation (BuildSys '21), November 17-18, 2021, Coimbra, Portugal. ACM, New York, NY, USA, 4 pages. https://doi.org/10. $1145 / 3486611.3491130$

\section{INTRODUCTION}

Despite progress on meeting sustainable development goals (SDGs) worldwide, over a billion people worldwide continue to have only sporadic access to electricity. This sporadic access translates to regular grid black-outs ranging from a few seconds to multiple hours, for entire neighborhoods or cities. This phenomenon is especially widespread in South Asia, where a combination of rapidly increasing energy demand, poor grid infrastructure and supply-side

Permission to make digital or hard copies of all or part of this work for personal or classroom use is granted without fee provided that copies are not made or distributed for profit or commercial advantage and that copies bear this notice and the full citation on the first page. Copyrights for components of this work owned by others than ACM must be honored. Abstracting with credit is permitted. To copy otherwise, or republish, to post on servers or to redistribute to lists, requires prior specific permission and/or a fee. Request permissions from permissions@acm.org.

BuildSys '21, November 17-18, 2021, Coimbra, Portugal

(c) 2021 Association for Computing Machinery.

ACM ISBN 978-1-4503-9114-6/21/11 . \$ \$15.00

https://doi.org/10.1145/3486611.3491130 constraints lead to a fragile power system. Households tackle the issue of frequent grid outages primarily by UPS systems which include battery storage [5].

In Pakistan, a large number of urban households have such UPS systems installed, which provide backup in the case of a grid outage. However, these systems also suffer from many shortcomings, including low efficiency and a naive charging regime which invariably creates additional demand peaks on the low-voltage distribution grid. However, while these backup systems provide a valuable service for the households, i.e. uninterrupted power supply, they can also be useful in helping the grid by realizing demand response programs in such buildings [4]. More specifically, this can help reduce the need for both expensive grid reinforcement and supplyside investments. By making demand more responsive and better matched to the supply, less excess generation capacity needs to be added. A thorough review of the algorithms that can be used to achieve this is provided in [1]. However, before demand response can be leveraged, the energy flexibility in the built environment must be identified [2].

The contribution of this paper is in the estimation of energy flexibility potential of residential buildings in Pakistan using real world data. This exploration is typically done in the context of a penalty signal sent to alter the behavior of the building, which is not present in this case. However, we show that it is possible to use grid outages as enforced energy flexibility requests. Using this forced flexibility event, we propose a method to estimate the flexibility of the buildings. Moreover, most existing energy flexibility studies focus on built environment in developed countries; this research shows how existing algorithms can be modified to bear also to the case of developing countries.

\section{METHODOLOGY AND SETUP}

\subsection{Setup}

We use the PRECON dataset to explore the energy flexibility of residential buildings in Lahore, Pakistan [6]. PRECON provides data for energy demand over a year (1 June, 2018 to 31 May, 2019) for 42 households, sampled on a per-minute basis. Additionally, the dataset includes meta-data about the buildings and their occupants. There is considerable diversity in the houses under consideration, which have been built between 1976 and 2015, and range from $63 \mathrm{~m}^{2}$ to over $3000 \mathrm{~m}^{2}$ in area. Sub-metered data for a number of important loads is also included in the dataset. This includes data for air conditioners, refrigerators and fans. It is important to note that most (93\%) of the buildings under consideration already have a UPS system installed to provide backup in case of a grid outage. Not all of these systems are sub-metered however. 


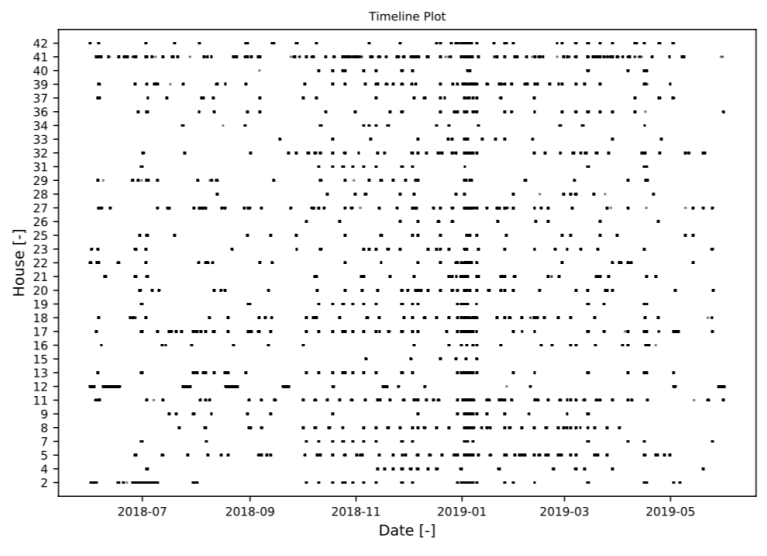

Figure 1: Timeline plot of estimated outages

Grid outages or black-outs are a recurring phenomenon in Pakistan. The situation in urban centers has improved considerably over the past few years due to massive supply-side investments, but as Fig. 2 shows there are still a number of outage events over the course of the year for which data is available. An important note here is that the dataset does not provide any explicit measure for when an outage occurs, the data recording simply returns no values for the duration of the outage. Hence, we plot load shedding events as times where the energy demand was below $5 \mathrm{~W}$ for at least half an hour. The median length for these outage events was around 1.5 hours, although the most frequent duration remained around one hour.

These outage events can be seen as an extreme form of flexibility activation, where the building occupants do not get to choose whether to participate or opt-out. Their only option is to shift or curtail their energy demand during the black-out event, according to their needs and the presence of battery storage. Therefore, during each outage event, the household energy demand can be (1) met during the outage event through the UPS system, (2) curtailed permanently, or (3) shifted to a later point in time. In reality, the UPS system is often dimensioned to meet only part of the energy demand, and therefore some energy is either curtailed (e.g. lighting in outdoor spaces) or shifted in time (e.g. white goods). In inertial loads such as space conditioning, there is likely to be a mix of both curtailment and shifting.

It is important to note that the dataset does not provide measurements for energy supplied by the UPS during the outage event. Therefore, it is not straightforward to calculate how much curtailment actually takes place. This must instead be estimated using excess UPS energy demand after the outage event, as we explain in subsequent sections. First, we discuss how to characterize flexibility in the residential context.

\subsection{Flexibility characterization}

Following the definition of energy flexibility from Annex 67 on energy flexible buildings, we utilize the concept of a Flexibility Function (FF), which allows us to consider the transient behaviour of buildings [3]. This is important when the flexibility potential is frequently activated so that the building is not in steady state

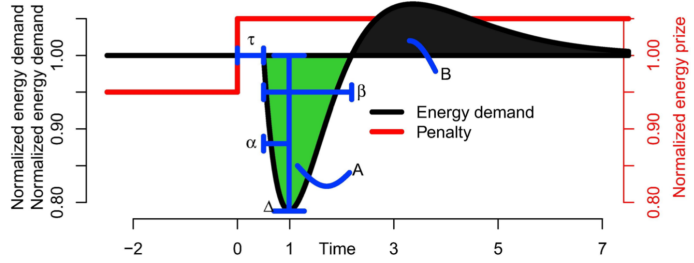

Figure 2: Flexibility characterization [3]

any more. The flexibility function assumes a penalty-aware smart building and an activation function, based on a penalty signal. The building's response to the activation function can be described using six different Flexibility Characteristics (FC), which we link to the present study as follows:

(1) A, Curtailed energy [kWh], is the difference between the predicted energy during the outage (i.e. the flexibility event) and the actual load during the outage (i.e. met with the UPS).

(2) $B$, Shifted energy $[\mathrm{kWh}]$, is the difference between the actual load after the outage and the predicted counterfactual load (i.e. in the case where there had been no outage). Since it is expected that demand will be higher after the outage due to shifted load, this parameter should be positive.

(3) $\Delta[\mathrm{kW}]$ is the maximum activation energy i.e. the power that is curtailed during the outage. In this case, the outage 'activates' total demand in the building, i.e. even what would be considered uncontrollable, such as lights, etc. in a normal automated demand response set-up.

(4) $\tau[\mathrm{h}]$ is the delay between the activation function and the building's response, which is zero in the case of the forced outage, as the curtailment is instantaneous.

(5) $\alpha[\mathrm{h}]$ is the time it takes to reach the maximum activation power, which is also zero in this case.

(6) $\beta[\mathrm{h}]$ is the total duration for which energy demand can be changed, i.e. the duration of the outage event in this case.

In this case therefore $A$ and $B$ are the only variables that still need to be estimated. Estimating these quantities requires a prediction for the energy demand during and after the outage event respectively. The methodology for making the forecasts is discussed in the next section, but first we look at the specificity of estimating the flexibility of the UPS system which we consider for demand response.

2.2.1 Energy consumption during the outage. The first step in flexibility characterization is to estimate the energy consumed by the household during the outage event. As this is not directly measured, it is approximated by the excess energy consumed by the UPS after the outage event to recharge the battery. Consequently, this is the difference between the actual UPS energy draw after the outage and the estimated UPS baseload (estimated as the seven-day rolling median of UPS power draw in normal conditions), while taking the (low) efficiency of the system into consideration as well.

2.2.2 Counterfactual energy consumption during the outage. The next step is to estimate the counterfactual case of baseline demand, i.e. how much energy would have been consumed during the outage 


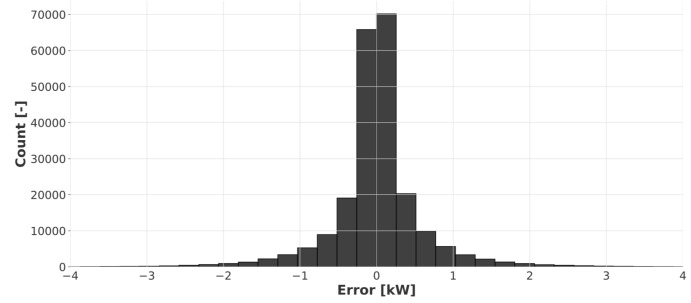

Figure 3: The residuals for the Prophet model are normally distributed, even though occasionally large forecast errors are made.

if there was no outage event (i.e. if flexibility was not activated). This is estimated as the predicted load during the outage event. The non-controllable component of this load is derived simply by subtracting the UPS baseload from the predicted load. This allows us to directly estimate the curtailed energy, $A$.

2.2.3 Energy consumption after the outage. Finally, we consider the excess energy demand after the outage event, excluding the UPS demand. This is the load that was shifted due to the forced flexibility activation, and is estimated as the difference between the predicted non-controllable load vs. the observed non-controllable load (i.e. the UPS power demand is excluded in both terms). This allows us to directly estimate the amount of shifted energy, $B$.

\subsection{Demand prediction}

Creating high resolution forecasts for residential building energy demand is a challenging problem. It is well known that stochastic human behaviour can lead to large errors in forecasts at this granular level. This is especially true for non-thermal loads, i.e. those which are not directly linked to ambient conditions. Nevertheless, for this research, we create forecasts for the energy demand in these buildings using two different techniques: a multilinear regression model that uses the partial autocorrelation function with the historical dataset for feature selection, and a time series prediction model with exogenous features that makes use of the open-sourced Facebook Prophet library. We use historical observations and weather data as inputs to the demand predictor, which produces as its output the electricity demand for a specified time horizon $t$. Optimal hyperparameters are selected using forward chaining, a cross-validation technique for time series data. Building meta-data is not used in the predictors, but is an interesting direction for future work. The multi-linear regression model is implemented as a recursive forecaster.

Both models perform as well as can be expected for residential data, with the Prophet model showing marginally better performance than the linear regression. The error distribution is shown in Fig. 3, while the relative mean absolute error (rMAE) of the Prophet model when compared to daily and weekly naive models is around 0.71 and 0.87 respectively. Consequently, we use the Prophet model for forecasting in subsequent estimation steps.

\begin{tabular}{lccccccccc}
\hline House & $\mathbf{2}$ & $\mathbf{5}$ & $\mathbf{7}$ & $\mathbf{8}$ & $\mathbf{1 1}$ & $\mathbf{1 3}$ & $\mathbf{1 9}$ & $\mathbf{2 0}$ & $\mathbf{3 1}$ \\
\hline \hline $\begin{array}{l}\text { Yearly UPS } \\
\text { demand [kWh] }\end{array}$ & 693 & 1462 & 1981 & 36 & 1125 & 1418 & 1843 & 4276 & 1211 \\
\hline $\begin{array}{l}\text { Ratio demand } \\
\text { UPS/Total [\%] }\end{array}$ & $19 \%$ & $26 \%$ & $22 \%$ & $1 \%$ & $24 \%$ & $29 \%$ & $17 \%$ & $71 \%$ & $11 \%$ \\
\hline Median power [W] & 44 & 123 & 205 & 1 & 102 & 165 & 155 & 471 & 120 \\
\hline Max power [kW] & 0.53 & 2.38 & 1.24 & 0.86 & 0.8 & 1.28 & 1.63 & 3.85 & 0.68 \\
\hline
\end{tabular}

Figure 4: Summary of the UPS energy demand data across nine households

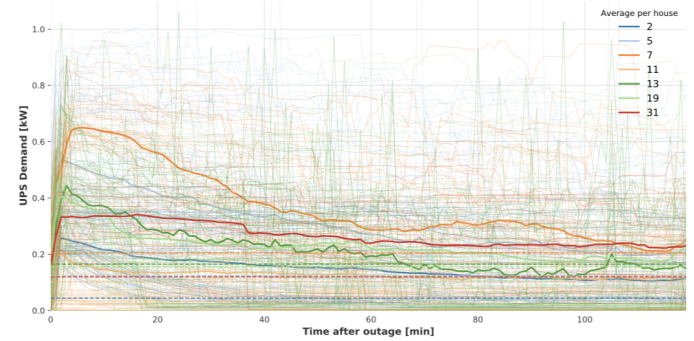

Figure 5: Profiles of energy demand by UPS systems in different households after outage events

\section{RESULTS}

Of the 42 households included in the PRECON dataset, there are nine which have sub-metered demand data for the UPS system. The energy demand for the different UPS systems is shown in Fig. 4, along with its max and median power over the entire year. The UPS systems tend to account for roughly $20 \%$ of the overall energy demand in the buildings on average, which is extremely high. This is primarily caused by the very high self-discharge rates of the UPS systems in most houses. There are two clear outliers. House 8 seems to make almost no use of the UPS, which means it was either installed or measured incorrectly, or that it had broken down during the measurement period. House 20 on the other hand has a UPS-demand that accounts for $71 \%$ of the total demand of the house. It seems highly likely that a measurement error has occurred due to improper installation of the smart-meter or because of a faulty setup of the UPS-system itself. The UPS-data from these two houses will not be taken into account in further analysis, leaving us with seven households for which data is available for the entire year.

Across these remaining households, the dataset contains 334 outage events over the entire year which we analyse in this section to estimate the relevant flexibility characteristics, $A$ and $B$. Fig. 5 shows the UPS charging behaviour for these households after all of these outage events (forced flexibility events). Immediately after the outage event, there is an increase in energy demand to recharge the UPS system. On average, within two hours, the UPS energy demand falls back to its median energy demand. Individual differences across households are quite pronounced as well: household 7 has a postoutage peak that is over three times the baseline, compared to household 2 and 13 where this difference is much smaller.

\subsection{Excess energy}

The excess energy demand is estimated as the UPS charging energy (excluding the self-discharge load) after an outage. An average of 


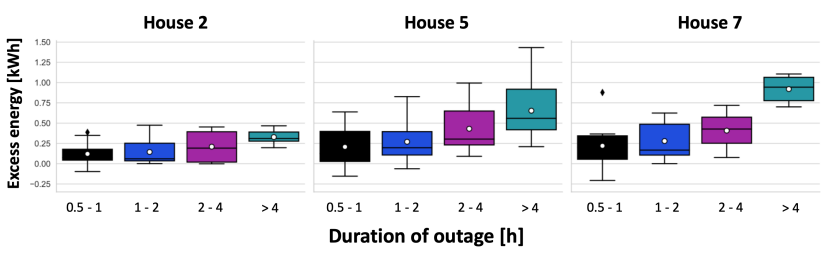

Figure 6: Effect of outage duration on excess UPS usage (excl. self-discharging) for three different households

$0.21 \mathrm{kWh}$ of charging energy is found for all households. The distribution is compact and skews to the right with a number of outliers around $1 \mathrm{kWh}$. Furthermore, using data from three households, Fig. 6 shows that the energy demand of the UPS after the outage event is a strong function of the duration of the load shedding event. This indicates that households either have batteries which do not usually run out of power during outage events (i.e. are overdimensioned) or that the households ration their energy usage during these events (i.e. curtail the load more heavily to preempt a longer than expected outage). This excess energy can be directly used as a flexibility source. This is of interest because, as Fig. 5 shows there is considerable additional load on the grid after outage events which may lead to elevated peak demand, further exacerbating issues for an already overloaded electric distribution network.

\subsection{Curtailed energy, $A$}

Fig. 7 shows the distribution of curtailed energy, $A$, for different outage events and houses. Using the amount of estimated energy consumed by the households and the predicted load during the outage, we estimate that between 0.4 and $1.4 \mathrm{kWh}$ of energy was curtailed on average per house per outage event. These are however positively skewed due to a number of outliers. The median curtailed energy of the different houses is thus smaller and ranges between 0.16 and $1.03 \mathrm{kWh}$ per household per outage, which is between $1.3 \%$ and $3.3 \%$ of the average daily consumption. This number is naturally larger for households with less energy demand or multiple outage events per day. While rather limited, the numbers nevertheless show that there is definitely some potential for demand response. At the same time, they also indicate that the UPS systems are meeting most of the energy requirements during the outage event since there is only limited curtailment.

\subsection{Shifted energy, $B$}

Fig. 7 shows the distribution of shifted energy, $B$, for the houses under consideration. When considering the total demand, the average shifted energy is close to zero, between $-0.38 \mathrm{kWh}$ and 0.13 $\mathrm{kWh}$. These values seem to be evenly distributed around zero. It would thus mean that after an outage event there is on average no, or very little, increased energy usage to compensate for the unavailability of loads during the outage. This can be explained by the non-shiftable nature of loads which are not powered by the UPS (e.g. lighting, television etc.). The shifting time may also be greater than that considered here. Finally, the prediction accuracy for the counterfactual demand may also have played a role here, even though it is not straightforward to ascertain this.
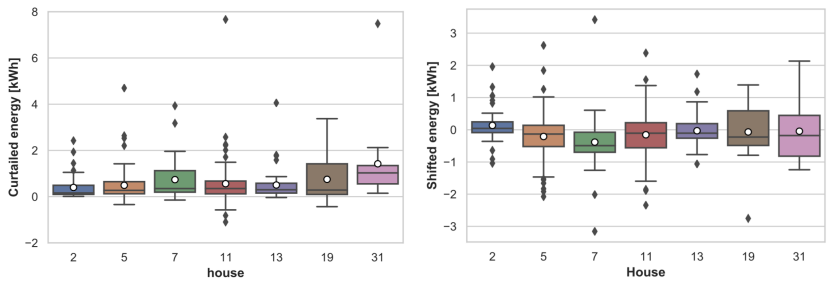

Figure 7: (Right) Curtailed energy as a result of the outage event; (Left) Shifted energy as a result of the outage event

\section{CONCLUSION}

Preliminary results presented in this paper indicate that forced outages in Pakistan produce a non-negative curtailment in household energy demand, even though energy shifting remains inconclusive. One reason for the limited energy shifting seems to be that most of the outage events are during winter months, while the primary shiftable load (i.e. air conditioner) is mostly in use during the summer months. These results also heavily depend on our ability to reasonably predict the counterfactual demand in case the outage would not have happened. For example, in 23 of the analysed cases, the curtailed energy is smaller than zero, which is not possible in real life. This means that there is some estimation error which can be reduced either by better forecasting models or through direct feedback from the households.

At the same time, the research points towards some exciting future directions. Foremost amongst these is the extension to other flexible loads. Furthermore, while the focus in this paper has been on quantifying energy flexibility, it is also important to leverage this flexibility in practice. The most obvious use case of energy flexibility on a building level is to charge and discharge the battery in a way that minimizes losses incurred due to its high self-discharge rate. At the grid level, it should be possible to utilize the energy flexibility of the UPS system to reduce the adverse effects of coordinated charging of UPS systems in a neighborhood. This latter use case can have a small negative side-effect on household utility i.e. it increases the risk of energy curtailment during a subsequent outage.

\section{REFERENCES}

[1] Christian Finck, Paul Beagon, John Claus, Thibault Pean, Pierre Vogler-Finck, Kun Zhang, and Hussain Syed Kazmi. 2018. Review of applied and tested control possibilities for energy flexibility in buildings. Review of applied and tested control possibilities for energy flexibility in buildings (2018).

[2] Søren Østergaard Jensen, Anna Marszal-Pomianowska, Roberto Lollini, Wilmer Pasut, Armin Knotzer, Peter Engelmann, Anne Stafford, and Glenn Reynders. 2017. IEA EBC annex 67 energy flexible buildings. Energy and Buildings 155 (2017), $25-34$.

[3] Rune Grønborg Junker, Armin Ghasem Azar, Rui Amaral Lopes, Karen Byskov Lindberg, Glenn Reynders, Rishi Relan, and Henrik Madsen. 2018. Characterizing the energy flexibility of buildings and districts. Applied energy 225 (2018), 175-182.

[4] Hussain Kazmi and Johan Driesen. 2020. Automated Demand Side Management in Buildings. In Artificial Intelligence Techniques for a Scalable Energy Transition. Springer, 45-76.

[5] Hussain Kazmi, Fahad Mehmood, Zhenmin Tao, Zainab Riaz, and Johan Driesen. 2019. Electricity load-shedding in Pakistan: Unintended consequences, opportunities and policy recommendations. Energy Policy 128 (2019), 411-417.

[6] Ahmad Nadeem and Naveed Arshad. 2019. Precon: Pakistan residential electricity consumption dataset. In Proceedings of the Tenth ACM International Conference on Future Energy Systems. 52-57. 